\title{
Padronização e avaliação histológica de um modelo experimental de lesão medular
}

\author{
Standardization and histological evaluation of an experimental model of spinal cord injury
}

\author{
José Ademar Villanova Junior ${ }^{\mathrm{I}}$ Rosangela Locatelli-Dittrich ${ }^{\mathrm{I}}$ Letícia Fracaro $^{\mathrm{II}}$ \\ Carmen Lúcia Kuniyoshi Rebelatto II Luis Guilherme Achcar Capriglione ${ }^{\mathrm{II}}$ \\ Paulo Roberto Slud Brofman'II Liv Cristina MiaraIII \\ Corinne de Alcântara Fernandes Nascimento ${ }^{\mathrm{III}}$
}

RESUMO

A lesão medular é incapacitante, irreversivel e de custo econômico e social elevado. Neste estudo, objetivou-se padronizar um modelo de lesão medular, que produza paraplegia, com o uso de cateter e avaliar histologicamente a efetividade da lesão para estudos com terapia celular. Foram realizadas as lesões medulares em ratos Wistar, utilizando-se o cateter Fogarty $n .3$ e compressão na região toracolombar (T8-T9) durante 5 minutos. Foram estudados três grupos: grupo A, animais controle sem lesão medular; grupo B, animais submetidos à lesão, utilizando-se $50 \mu \mathrm{L}$ de compressão; grupo $C$, animais submetidos à lesão, utilizandose $80 \mu \mathrm{L}$ de compressão. Foi realizada avaliação motora pela aplicação da escala $B B B$, antes da compressão, após recuperação anestésica, 24 e 72 horas depois da compressão e sete dias após a compressão. Após o sétimo dia da lesão, os animais foram submetidos à eutanásia, foi feita a retirada da medula espinhal, figado e rins e realizada a análise histológica com a coloração hematoxilina-eosina. A mortalidade variou entre os grupos, com $0 \%$ no grupo $A, 38,5 \%$ no B e $48 \%$ no C. Nesses dois últimos grupos, a causa da morte foi edema pulmonar neurogênico, confirmado clinica e histologicamente. As medulas espinhais histologicamente apresentaram diferentes graus de edema, congestão vascular e hemorragia, enquanto que os figados e os rins apresentaram diferentes graus de congestão vascular e necrose. Em relação à recuperação dos movimentos, no grupo A, verificou-se $100 \%$ de escore 21 ; no $B, 25 \%$ de escore $21 ; 37,5 \%$ de escore 11 ; e $37,5 \%$ de escore 0; enquanto, no grupo C, verificou-se $100 \%$ de escore 0 . Conclui-se que o procedimento realizado utilizando-se $80 \mu \mathrm{L}$ de solução salina para preencher o balão do cateter foi mais eficiente, apesar de maior mortalidade, pois apresentou maior porcentagem de animais com lesão completa (paraplegia).

Palavras-chave: modelo de lesão medular, paraplegia, estudo histológico, edema pulmonar neurogênico.

\section{ABSTRACT}

Spinal cord injury is disabling, irreversible and with high economic and social cost. This study aimed to standardize a model of spinal cord injury to induce paraplegia, with a catheter and to evaluate the effectiveness of the histological lesion for further studies with cell therapy. Cord lesions were performed in Wistar rats using the Fogarty catheter $n .3$ and compression in the thoracolumbar region (T8-T9) for 5 minutes. We studied three groups: A control group without spinal cord injury, B group subjected to $50 \mu \mathrm{L}$ compression injury, C group with animals subjected to $80 \mu \mathrm{L}$ compression injury. Motor evaluation was performed by applying the $B B B$ scale, before compression, after recovery from anesthesia, 24 and 72 hours after compression and 7 days after compression. At the seventh day after injury, the animals were euthanized. The spinal cord, liver and kidneys were removed and a histological analysis was performed with hematoxylin-eosin staining. Mortality varied among groups, it was $0 \%$ in group A, $38.5 \%$ in group B and $48 \%$ in group $C$. In the latter two groups the cause of death was neurogenic pulmonary edema, clinically and histologically confirmed. Histologically the spinal cord showed different degrees of edema, hemorrhage and vascular congestion, while the liver and kidneys showed different degrees of vascular congestion and necrosis. Regarding movement recovery, in group $A$ it was found a $100 \%$ score 21, in group B 25\% of score 21, 37.5\% score 11 and $37.5 \%$ of score zero, whereas in group $C$ there was a $100 \%$ of score zero. It is concluded that the procedure performed using $80 \mu \mathrm{L}$ of saline to fill the balloon catheter was more efficient because, although the higher percentage of mortality, it induced a higher percentage of animals with complete injury (paraplegia).

Key words: spinal cord injury model, paraplegia, histology, neurogenic pulmonary edema.

\footnotetext{
'Programa de Pós-graduação em Ciências Veterinárias, Universidade Federal do Paraná (UFPR), Campus Curitiba, Rua dos Funcionários, 1540, 80035-050, Curitiba, PR, Brasil. E-mail: jose.villanova@pucpr.br.*Autor para correspondência.

${ }^{\text {IIN }}$ úcleo de Tecnologia Celular, Pontifícia Universidade Católica do Paraná (PUCPR), Curitiba, PR, Brasil.

IIIUnidade Hospitalar para Animais de Companhia, PUCPR, São José dos Pinhais, PR, Brasil. 


\section{INTRODUÇÃO}

A lesão medular pode resultar em incapacidade sensitiva e motora permanente e seu tratamento ainda permanece um desafio, tanto para a medicina humana como veterinária (ARIAS et al., 2007), pois, além dos neurônios não se regenerarem (JEFFERY et al., 2001), não há consenso sobre o tratamento ideal (OLBY \& JEFFERY, 2007). De acordo com FITZMAURICE (2011), não existe cura farmacológica para danos à medula espinhal.

Os estudos que envolvem modelos experimentais de lesão medular são fundamentais para a compreensão dos mecanismos primários e secundários de lesão tecidual, decorrentes do trauma raquimedular. Dados de estudos experimentais mostraram que distúrbios ocorridos em cobaias podem ser extrapolados a seres humanos, se as suas devidas proporções anatômicas, fisiológicas e metabólicas forem respeitadas (MEYER et al., 2003).

Há um século, ALLEN (1911) foi o primeiro a padronizar lesão experimental pela técnica de queda de esfera metálica com peso preestabelecido sobre a medula espinhal de cães. TARLOV et al. (1953) introduziram a técnica de compressão medular em cães por implantação de um balão inflável no espaço extradural. O desenvolvimento de balões infláveis menores tornou possível estudar tais modelos em animais de laboratório (RIVLIN \& TATOR, 1978). Guner \& Young em 1992 desenvolveram o Impactor NYU, respeitando os preceitos de Allen (MEYER et al., 2003), porém as variações nos resultados, devido à melhora motora espontânea parcial (SOUZA et al., 2009) ou total (ŠEDÝ et al., 2009), após alguns dias de lesão medular, justificam mais estudos que visem a um modelo mais fidedigno.

Os objetivos deste estudo foram padronizar um modelo de lesão medular com cateter que produza paraplegia e avaliar histologicamente a efetividade da lesão para estudos com terapia celular e suas possíveis implicações.

\section{MATERIAL E MÉTODOS}

Animais

Foram utilizados 41 ratos Wistar fêmeas adultos jovens, com média de 20 semanas de idade e peso variando de 250 a $270 \mathrm{~g}$.

Os animais foram mantidos em gaiolas coletivas com 2 ou 3 animais em cada uma delas, com água e ração ad libitum. O biotério dispôs de controle de temperatura $\left(22^{\circ} \mathrm{C} \pm 2{ }^{\circ} \mathrm{C}\right)$, umidade $(45 \% \pm 15 \%)$, ventilação (exaustores superiores e inferiores) e de ciclos luminosos de 12/12h (lâmpada fluorescente).

Indução do trauma medular

Os animais receberam analgesia preemptiva com citrato de fentanila $\left(0,3 \mathrm{mg} \mathrm{kg}^{-1}\right.$ IM) e sedação com diazepan $\left(2,5 \mathrm{mg} \mathrm{kg}^{-1} \mathrm{IP}\right)$. Foram anestesiados com isofluorano em circuito anestésico com oxigênio $100 \%$. Após a tricotomia e antissepsia, foi realizada incisão de $5,0 \mathrm{~cm}$ na linha média dorsal, tendo como referência os processos espinhosos da oitava vértebra torácica (T8) à primeira vértebra lombar (L1). O tecido subcutâneo foi incisado, seguido do afastamento subperiosteal da musculatura paravertebral, com uso de lâmina de bisturi no⒒

A décima primeira vértebra torácica (T11) foi identificada por contagem dos arcos costais. Nela foi realizada laminectomia e, em seguida, foi inserido e conduzido cranialmente o balão do cateter de Fogarty no3 no espaço epidural até os segmentos medulares T8-T9.

Acompressão medular foi por preenchimento do balão durante 5 minutos. Antes e logo após a compressão medular, a integridade do cateter foi testada fora do animal e, em caso de ruptura do balão, nova compressão de cinco minutos era realizada.

A aproximação da musculatura foi realizada com fio vicryl ${ }^{\circledR} 4-0$, em padrão de sutura ancorada de Ford, posteriormente foi realizada a dermorrafia com mononáilon 3-0, seguindo o mesmo padrão de sutura. Ao término do procedimento cirúrgico, foi administrada enrofloxacina $\left(10 \mathrm{mg} \mathrm{kg}^{-1}\right)$ por via intramuscular.

Após a recuperação anestésica, foi realizada analgesia com morfina ( $5 \mathrm{mg} \mathrm{kg}^{-1} \mathrm{SC}$ q.i.d), por três dias. Diariamente, em intervalos de 6 horas, foram realizados esvaziamentos manuais da bexiga urinária.

\section{Grupos}

Após randomização, os animais foram identificados e divididos aleatoriamente em três grupos: grupo A (controle) - três ratas submetidas à laminectomia em T11, e introdução do balão do cateter no canal vertebral até o nível de T8-T9, sem ser preenchido; grupo B: 13 ratas submetidas a trauma medular com $50 \mu \mathrm{L}$ de volume de solução salina, utilizada para preencher o balão; e grupo C: 25 ratas submetidas a trauma medular com volume de preenchimento de $80 \mu \mathrm{L}$.

Avaliação motora pós-lesão

Os animais foram observados antes da compressão, após recuperação anestésica, 24 horas, 
72 horas e sete dias após a compressão, em uma caixa plástica sem barreiras para a avaliação da atividade motora voluntária dos membros pélvicos, seguindo escala de BBB (BASSO et al., 1995). A escala varia entre 0 e 21 pontos e reflete as condições locomotoras do animal, em que escore 0 representa paralisia total do membro e o escore 21 atividade locomotora normal.

\section{Eutanásia e necropsia dos animais}

No 7ํ dia pós-trauma, os animais foram submetidos à eutanásia sob analgesia preemptiva com fentanil ( $\left.0,3 \mathrm{mg} \mathrm{kg}^{-1} \mathrm{IM}\right)$ e sedação com diazepan $\left(2,5 \mathrm{mg} \mathrm{kg}^{-1} \mathrm{IP}\right)$. Foram anestesiados com isofluorano em circuito anestésico com oxigênio $100 \%$, após incisão de pele e ressecção do esterno, foi feita a canulação do ápice do ventrículo esquerdo com agulha fina e pequena incisão na aurícula direita.

Foi conectado o sistema de infusão à agulha e infundidos por bomba infusora $150 \mathrm{ml}$ de solução salina isotônica, seguida de $350 \mathrm{ml}$ de paraformaldeído a 4\%. Em seguida, foi realizada necropsia e coleta de medula espinhal, pulmões, fígado e rins.

Análises histopatológicas de secções de medula espinhal, pulmões, fígado e rins

Os pulmões, fígados, rins e as regiões de medula espinhal que sofreram compressão dos animais dos grupos A, B e C, e dos que foram a óbito durante ou após compressão medular, foram fixados em formalina

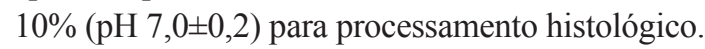

Análise estatística

Os resultados obtidos foram analisados estatisticamente pelos softwares Graphpad Prism v.5, SPSS 15.0 para Windows.

\section{RESULTADOS}

Avaliação motora pós-lesão

$\mathrm{Na}$ avaliação de recuperação dos movimentos, verificou-se, no grupo A, $100 \%$ de escore 21 ; no grupo B, $25 \%$ de escore $21,37,5 \%$ de escore 11 e $37,5 \%$ de escore 0 ; no grupo $\mathrm{C}$, verificouse $100 \%$ de escore 0 (Tabela 1 ).

Aspectos macroscópicos e microscópicos da zona de medula espinhal lesionada

Nos grupos B e C, a compressão medular causou zona hemorrágica com achatamento de parênquima (Figura 1). Microscopicamente, nesses animais, nas áreas da medula espinhal com compressão, verificou-se diminuição do número de neurônios, hemorragia, vacuolização, e malacia do parênquima medular (Figura 2).

Mortalidade durante e após compressão medular

Não ocorreu mortalidade no grupo A; no grupo $\mathrm{B}$, foi de $38,45 \%$ (sendo quatro óbitos durante a compressão e um após $24 \mathrm{~h}$ ); no grupo C, foi de $48 \%$ (sendo nove casos durante a compressão, um após $24 \mathrm{~h}$ e dois após 48h).

Antes da parada cardíaca, ocorreu taquicardia seguida de bradicardia, e bradipneia seguida de apneia. Epistaxe e hemoptise foram observadas em todos os animais que foram a óbito. Devido à manifestação clínica, aos resultados da necropsia e análises histopatológicas, conclui-se que o óbito ocorreu por edema pulmonar neurogênico (Figura 3).

Análises histopatológicas dos pulmões, fígado e rins dos animais que foram a óbito durante ou após compressão medular

Tabela 1 - Resultados referentes aos escores obtidos pelos animais, na Escala BBB, nos diversos momentos avaliados neste estudo.

\begin{tabular}{|c|c|c|c|c|c|c|}
\hline Grupo experimental & & & & & & Valor de $\mathrm{p}^{*}$ \\
\hline & Pré-teste & Após recuperação anestésica & $24 \mathrm{~h}$ & $72 \mathrm{~h}$ & 7 dias & \\
\hline Grupo A $(n=3)$ & $21 \pm 0 \mathrm{a} / \mathrm{a}$ & $21 \pm 0 \mathrm{a} / \mathrm{a}$ & $21 \pm 0 \mathrm{a} / \mathrm{a}$ & $21 \pm 0 \mathrm{a} / \mathrm{a}$ & $21 \pm 0 \mathrm{a} / \mathrm{a}$ & 1,00 \\
\hline Grupo B $(n=8)$ & $21 \pm 0 \mathrm{a} / \mathrm{a}$ & $9,35 \mathrm{~b} / \mathrm{b}$ & $9,35 \mathrm{~b} / \mathrm{b}$ & $9,35 \mathrm{~b} / \mathrm{b}$ & $9,35 \mathrm{~b} / \mathrm{b}$ & 0,0017 \\
\hline Grupo $C(n=13)$ & $21 \pm 0 \mathrm{a} / \mathrm{a}$ & $0 \pm 0 \mathrm{c} / \mathrm{a}$ & $0 \pm 0 \mathrm{c} / \mathrm{a}$ & $0 \pm 0 \mathrm{c} / \mathrm{a}$ & $0 \pm 0 \mathrm{c} / \mathrm{a}$ & 1,00 \\
\hline Valor de $\mathrm{p}$ (entre grupos)** & 1,00 & 0,001 & 0,001 & 0,001 & 0,001 & \\
\hline
\end{tabular}

Os valores estão expressos em média \pm erro padrão da média.

* Teste ANOVA de uma via de medidas repetitivas, seguido pelo pós-teste de Tukey.

** Teste ANOVA de uma via, seguido pelo pós-teste de Tukey.

Letras diferentes indicam diferença significativa entre os grupos/momentos (pós-teste de Tukey, $\mathrm{P}<0,05$ ). 


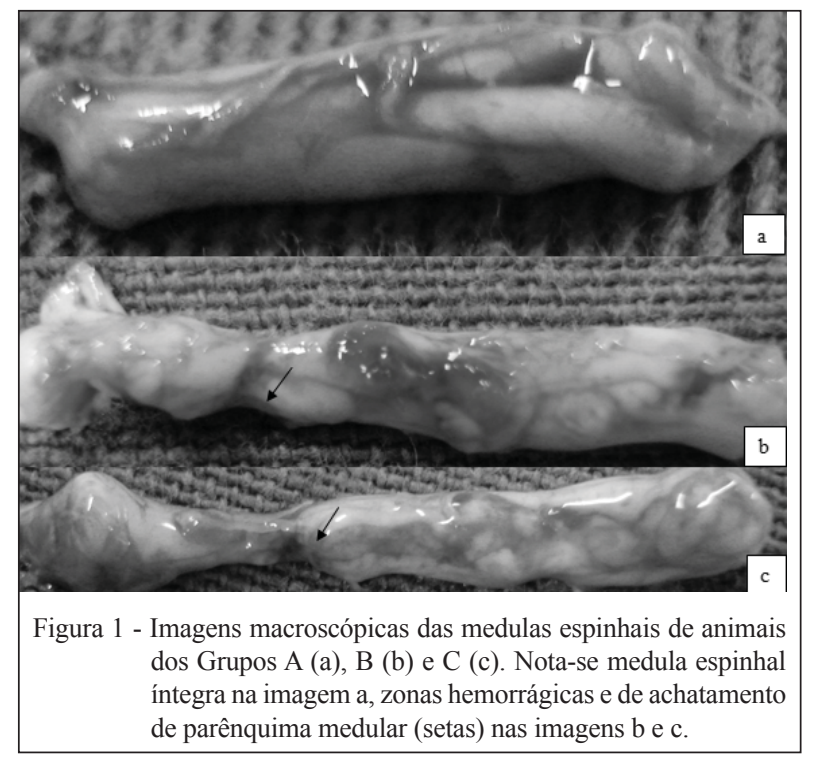

Os exames histopatológicos dos pulmões revelaram edema pulmonar de grau leve a moderado, acentuada congestão vascular e moderada hemorragia, sem áreas de necrose (Figura 4).

No fígado, observou-se congestão vascular de grau moderado a severo, necrose leve e ausência de edema e de hemorragia. Nos rins, ocorreu congestão vascular e necrose moderada, sem edema e hemorragia.

\section{DISCUSSÃO}

No presente estudo, foi utilizado o citrato de fentanila como agente analgésico único no período pré-operatório. Ele pode causar rigidez muscular, comprometendo particularmente os músculos torácicos e, durante a indução da anestesia, pode também atingir os movimentos musculares esqueléticos de vários grupos nas extremidades, pescoço e globo ocular. Esses efeitos estão relacionados com a dose e a velocidade de injeção e a incidência pode ser evitada através das seguintes medidas: injeção intravenosa lenta, uso de benzodiazepínicos na pré-medicação ou uso de relaxantes neuromusculares. Neste estudo, foi realizada a infusão lenta e a associação com um benzodiapénico, porém a ação do citrato de fentanila é curta (ALVES et al., 2010). Baseado no estudo de DAMY et al. (2010), recomenda-se a substituição desse fármaco por buprenorfina $0,02-0,5 \mathrm{mg} \mathrm{kg}^{-1} \mathrm{SC}$, IP ou IM. O tempo de analgesia obtido, segundo esses autores, é de 6-12h, tempo suficiente para o acesso cirúrgico, compressão medular, oclusão de planos anatômicos e retorno anestésico.

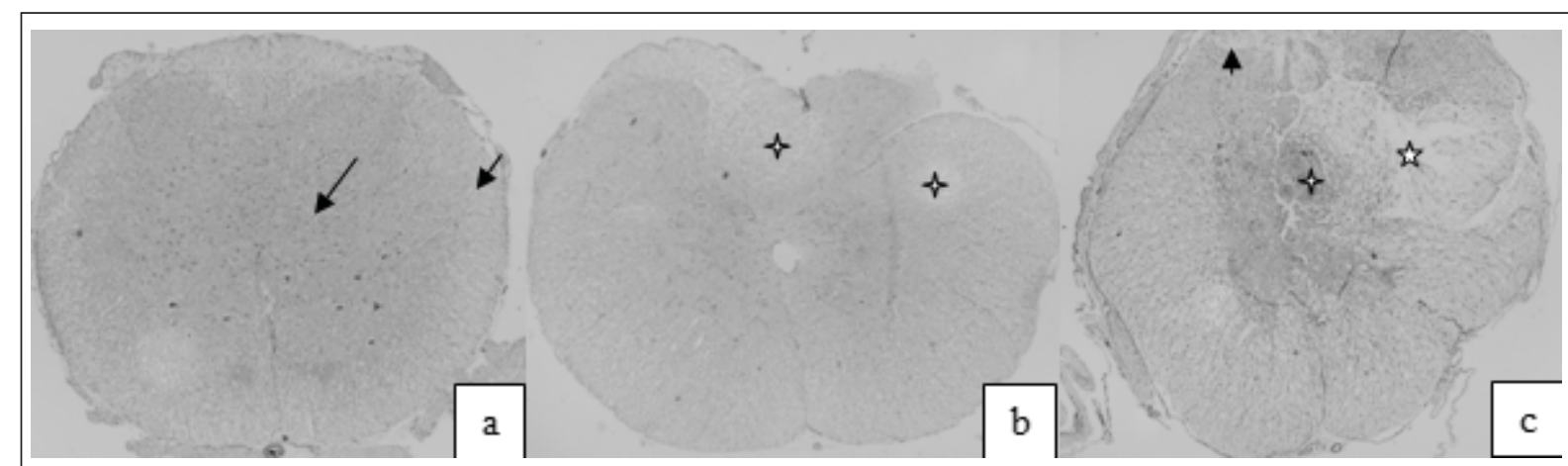

Figura 2 - Cortes histológicos do epicentro das compressões medulares dos Grupos A (a), B (b) e C (c). Nota-se na imagem a preservação das substâncias branca (seta menor) e cinzenta (seta maior). Na imagem b nota-se áreas com necrose e vacuolização (asteriscos), enquanto que na imagem c nota-se hemorragia (asterisco), necrose e vacuolização (seta), diminuição de neurônios motores e ruptura do parênquima medular (estrela). 


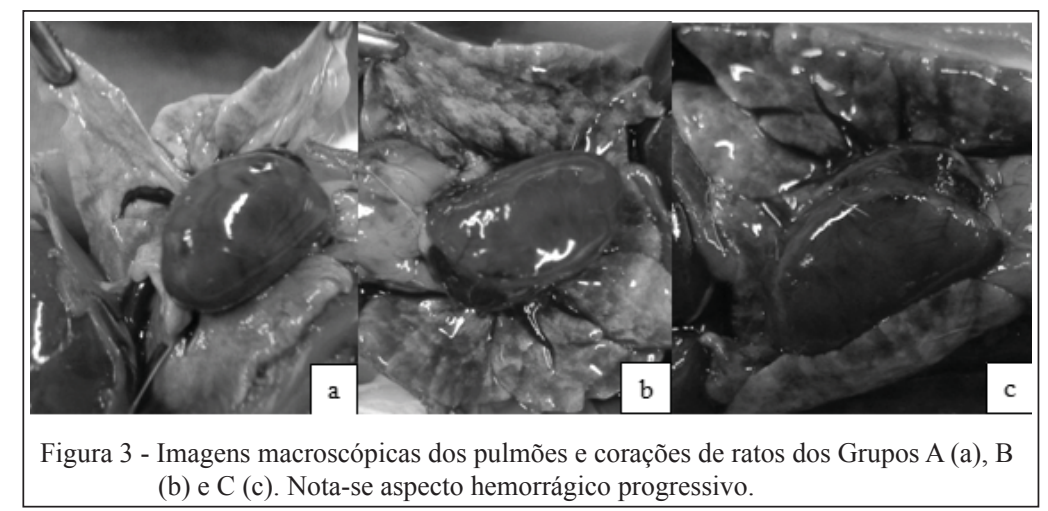

A avaliação motora utilizada neste estudo mostrou que a laminectomia e introdução do cateter vazio no espaço epidural foi incapaz de causar déficit motor, pois todos os animais do Grupo A tiveram escore BBB de 21. Nos animais do grupo C, o déficit motor ocorreu devido ao volume de compressão, uma vez que $100 \%$ tiveram escore BBB de 0 . No grupo $\mathrm{B}$, foi observada grande variação de déficit motor, creditada à incapacidade da compressão de $50 \mu \mathrm{L}$ causar paralisia total por lesão primária, decorrente das forças que causam dano mecânico instantaneamente após o evento traumático. ŠEDÝ et al. (2009) observaram que, na compressão de $15 \mu \mathrm{L}$, há déficit motor de escore 9-11 na escala BBB. Com 5 e $10 \mu \mathrm{L}$, esses autores observaram melhora espontânea progressiva e completa da motricidade, escore 21, tornando esse volume não recomendável para produzir lesão medular.

No presente estudo, não foram verificadas variações motoras e o quadro após recuperação anestésica foi mantido até o dia da eutanásia, resultados que diferem dos de SOUZA et al. (2009), que estudaram as consequências da lesão por contusão da medula espinhal, associada ao estreitamento do canal vertebral, no comportamento motor de ratos, avaliando-se o efeito do tempo para descompressão na recuperação neurológica dos animais. Excetuando o grupo controle, perceberam melhora parcial espontânea e progressiva em todos os grupos, as análises motoras por eles realizadas ocorreram 1, 3 e 7 dias após a compressão.

A lesão primária nos animais deste estudo foi induzida pela contusão da medula espinhal, produzida pela ruptura do parênquima medular, lesão vascular e hemorragia, quadro observado em todas as análises histológicas dos animais do Grupo C. Essas lesões se propagam devido à ativação de eventos bioquímicos, levando à necrose e lesão excitotóxica (ARIAS et al., 2007; PARK et al., 2010). LEAL FILHO et al. (2005) observaram hemorragia

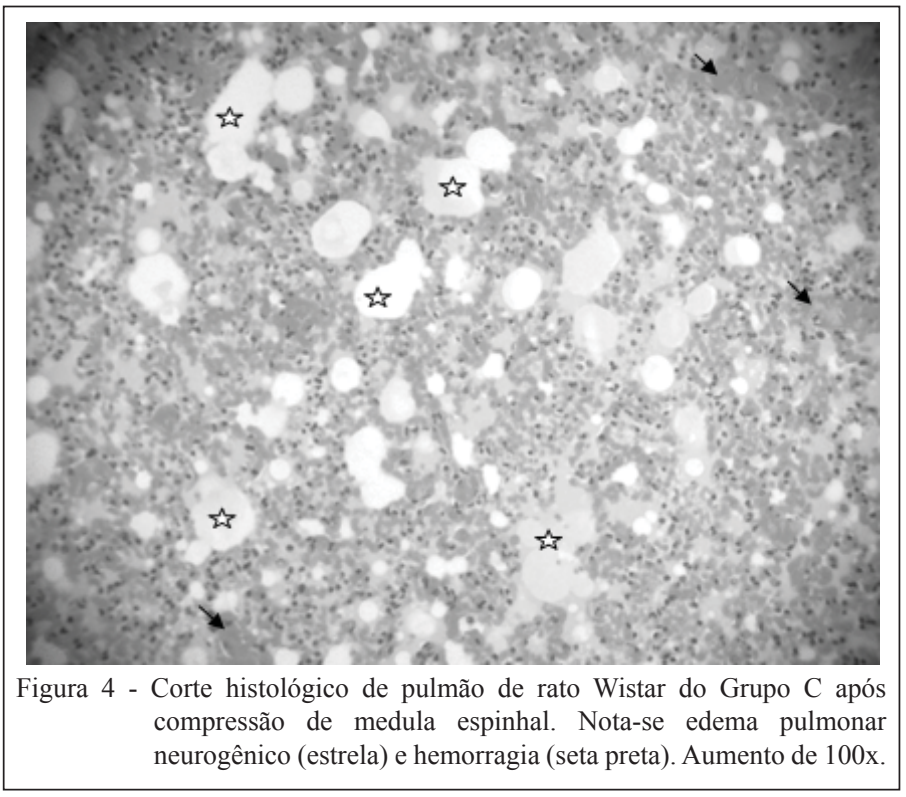

Ciência Rural, v.44, n.6, jun, 2014. 
e discreta descontinuidade unilateral de parênquima medular, após compressão de $20 \mu \mathrm{L}$, durante um minuto, resultado que não foi correlacionado com a análise motora. Esses autores verificaram edema pulmonar e congestão renal e hepática, resultados também observados no presente estudo.

A mortalidade observada neste estudo, pela insuflação do balão e consequente compressão medular, está associada à grande ativação simpática, que causou aumento de pressão arterial, ativação barorreflexa e redistribuição de sangue dos vasos esplâncnicos para o leito vascular pulmonar, desencadeando edema pulmonar neurogênico (ŠEDÝ et al., 2009). As porcentagens de mortalidade nos grupos $\mathrm{B}$ e $\mathrm{C}$ foram próximas; tais manifestações clínicas foram também observadas por LEAL FILHO et al. (2005) e ŠEDÝ et al. (2009), com cateter mais fino, menor insuflação do balão e menor tempo de compressão.

As lesões medulares ainda são consideradas intratáveis e incuráveis (ARIAS et al., 2007), não há cura farmacológica (FITZMAURICE, 2011) e, apesar das evidências científicas favoráveis ao uso de células-tronco em doenças neurológicas, consideráveis avanços necessitam ser feitos (PAULA et al., 2005; PEREIRA, 2008).

A validação de modelo experimental de lesão medular reprodutível, de fácil feitura e pouco dispendioso, como realizado neste estudo, abre perspectivas para a realização de mais estudos que envolvam lesão medular e terapia celular.

\section{CONCLUSÃO}

O modelo de lesãomedular com compressão de $80 \mu \mathrm{L}$ para insuflar o balão do cateter é válido, pois resultou em paraplegia em todos os animais e não houve melhora espontânea e progressiva, apesar de acarretar índice médio de mortalidade.

\section{COMITÊ DE ÉTICA E BIOSSEGURANÇA}

$$
\text { REGISTRO DO PROJETO: } 601 \text {-APROVADO 1ํㅗㄹ versão. }
$$

\section{REFERÊNCIAS}

ALLEN, A.R. Surgery of experimental lesion of spinal cord equivalent to crush injury of fracture dislocation of spinal column. Preliminary report. Journal of the American Medical Association, v.57, p.878-880, 1911. Disponível em: $<$ http://jama. jamanetwork.com/article.aspx?articleid $=448138>$. Acesso em: 10 fev. 2013. doi: 10.1001/jama.1911.04260090100008.

ALVES, H.N.C. et al. A Anesthesia with intraperitoneal propofol, medetomidine, and fentanyl in rats. Journal of the American Association for Laboratory Animal Science, v.49, n.4, p.454459, 2010. Disponível em: <http://www.ncbi.nlm.nih.gov/pmc/
articles/PMC2919186/>. Acesso em: 10 fev. 2013. doi: 10.1258/ la.2008.007036.

ARIAS, M.V.B. et al. Trauma medular em cães e gatos: revisão da fisiopatologia e do tratamento médico. Semina: Ciências Agrárias, v.28, n.1, p.115-134, 2007. Disponível em: $<$ http://www.uel.br/revistas/uel/index.php/semagrarias/article/ view/2568/2233>. Acesso em: 10 fev. 2013

BASSO, D.M. et al. A sensitive and reliable locomotor rating scale for open field testing in rats. Journal of Neurotrauma, v.12, n.1, p.1-21,. 1995. Disponível em: <http://online.liebertpub.com/ doi/abs/10.1089/neu.1995.12.1>. Acesso em: 10 fev. 2013. doi: 10.1089/neu.1995.12.1.

DAMY, S.B. etal. Aspectos fundamentais da experimentação animal - aplicações em cirurgia experimental. Revista da Associação Médica Brasileira, v.56, n.1, p.103-111, 2010. Disponível em: $<\mathrm{http}$ ://www.scielo.br/pdf/ramb/v56n1/24.pdf $>$. Acesso em: 10 fev. 2013. doi: 10.1590/S0104-42302010000100024.

JEFFERY, N. et al. Bridging the divide: spinal cord repair by cellular transplantation-from research laboratory to therapeutic application. Journal of Small Animal Practice, v.42, p.428432, 2001. Disponível em: <http://onlinelibrary.wiley.com/ doi/10.1111/j.1748-5827.2001.tb02495.x/pdf>. Acesso em: 10 fev. 2013. doi: 10.1111/j.1748-5827.2001.tb02495.x.

FITZMAURICE, S.N. Neurologia em pequenos animais. Rio de Janeiro: Elsevier, 2011. 332p.

LEAL FILHO, M.B. et al. Hemodynamic parameters and neurogenic pulmonary edema following spinal cord injury - an experimental model. Arquivos de Neuropsiquiatria, v.63, n.4, p.990-996, 2005. Disponível em: <http://www.scielo.br/pdf/anp/ v63n4/a16v63n4.pdf>. Acesso em: 10 fev. 2013. doi: 10.1590/ S0004-282X2005000600016.

MEYER, F. et al. Alterações vesicais na lesão medular experimental em ratos. Acta Cirúrgica Brasileira, v.18, n.3, p.203-208, 2003. Disponível em: <http://www.scielo.br/pdf/acb/ v18n3/a07v18n3.pdf>. Acesso em: 10 fev. 2013. doi: 10.1590/ S0102-86502003000300007.

OLBY, N.; JEFFERY, N. Patogenia das doenças do sistema nervoso central. In: SLATTER, D. Manual de cirurgia de pequenos animais. 3.ed. São Paulo: Manole, 2007. Cap.77, p.1132-1147.

PARK, W.B. et al. The effect of mesenchymal stem cell transplantation on the recovery of bladder and hindlimb function after spinal cord contusion in rats. BioMed Central Neuroscience, v.11, p.119-130, 2010. Disponível em: <http:// www.biomedcentral.com/content/pdf/1471-2202-11-119.pdf>. Acesso em: 10 fev. 2013. doi: 10.1186/1471-2202-11-119.

PAULA, S. et al. O potencial terapêutico das células-tronco em doenças do sistema nervoso. Scientia Medica, v.15, n.4, p.263269, 2005. Disponível em: <http://revistaseletronicas.pucrs.br/ojs/ index.php/scientiamedica/article/viewFile/1578/1181>. Acesso em: 10 fev. 2013

PEREIRA, L.V. A importância do uso das células-tronco para a saúde pública. Ciência \& Saúde Coletiva, v.13, p.714, 2008. Disponível em: <http://www.scielo.br/pdf/csc/

Ciência Rural, v.44, n.6, jun, 2014. 
v13n1/01.pdf>. Acesso em: 10 fev. 2013. doi: 10.1590/S141381232008000100002 .

RIVLIN, A.S.; TATOR, C.H. Effect of duration of acute spinal cord compression in a new acute cord injury model in the rat. Surgical Neurology, v.10, n.1, p.38-43, 1978. Disponível em: $<$ http://www.ncbi.nlm.nih.gov/pubmed/684604>. Acesso em: 10 fev. 2013. doi: 10.1007/978-1-61779-301-1_18.

ŠEDÝ, J. et al. Rapid but not slow spinal cord compression elicits neurogenic pulmonary edema in the rat. Physiological Research, v.58, p.269-277, 2009. Disponível em: <http://www.biomed.cas. cz/physiolres/pdf/58/58_269.pdf $>$. Acesso em: 10 fev. 2013.
SOUZA, A.S. et al. Influência do estreitamento do canal vertebral e do tempo para a descompressão na recuperação locomotora de ratos. Coluna/Columna, v.8, n.2, p.117-126, 2009. Disponível em: <http://www.scielo.br/pdf/coluna/ v8n2/04.pdf $>$. Acesso em: 10 fev. 2013. doi: 10.1590/S180818512009000200004

TARLOV, I.M. et al. Spinal cord compression studies: I. Experimental techniques to produce acute and gradual compression. AMA Archives of Neurology \& Psychiatry, v.70, p.813-818, 1953. Disponível em: <http://archneurpsyc.jamanetwork.com/ article. $a s p x$ ?articleid $=651687>$. Acesso em: 10 fev. 2013. doi: 10.1001/archneurpsyc.1953.02320360128010.

Ciência Rural, v.44, n.6, jun, 2014. 